\title{
Antiaging, photoprotective, and brightening activity in biorevitalization: a new solution for aging skin
}

\author{
Adele Sparavigna \\ Beatrice Tenconi \\ lleana De Ponti \\ Derming, Clinical Research and \\ Bioengineering Institute, Monza, Italy
}

This article was published in the following Dove Press journal:

Clinical, Cosmetic and Investigational Dermatology

10 February 2015

Number of times this article has been viewed
Background: Age-related changes in the dermis can be considered the result of intrinsic factors and the consequence of environmental damage, mainly due to ultraviolet (UV) radiation from the sun (responsible for skin photoaging). The great versatility of the mesotherapy "biorevitalization" lies in the synergy between different biological effects of the active injected substances, which treats the skin in a more complete way. Several studies about biorevitalization efficacy showed good results. To date, however, objective results supported by instrumental evaluation are very sparse.

Purpose: This study evaluated the efficacy of an injectable solution (32 $\mathrm{mg}$ of hyaluronic acid plus an antiaging antioxidant complex consisting of vitamins, minerals, and amino acids) in the treatment of skin aging and photoaging.

Methods: A total of 64 female volunteers (37-60 years) underwent four sessions of biorevitalization at 3-week intervals, involving multiple injections in the face (external corner of the eye and cheek), neck, décolletage, and back of the hands. The esthetic result was assessed at baseline and after 6, 9, and 12 weeks, and was established through the use of clinical and instrumental evaluations, supported by photographic documentation. Additionally, a phototest was performed to assess the effect of biorevitalization treatment on UVB-induced erythema.

Results: Instrumental assessment showed, as early as after the second biorevitalizing treatment, the antiaging efficacy of the tested product; there was a clinical and statistically significant improvement of profilometric parameters, skin brightness, pigmentation, and deep skin hydration. The study product induced a statistically significant decrease of the visual score of the UVB-induced erythema compared with baseline, which was statistically different from placebo.

Conclusion: The study confirmed the well-known efficacy of biorevitalization in skin rejuvenation. The positive difference between deep and superficial skin hydration registered at the end of the trial suggested improved skin moisture retention of the stratum corneum. Furthermore, the obtained results suggest that the injected product could intervene at different moments of the skin pigmentation process by activating an intrinsic photoprotective mechanism and improving skin pigmentation quality. It may be that these processes employ common mechanisms in which antioxidants could play a pivotal role. This last hypothesis deserves further investigation.

Keywords: hyaluronic acid, antioxidants, photoaging, senile lentigo

\section{Introduction}

\section{Background}

The increasing concern to maintain a youthful appearance is driving the growth of new dermatological procedures for treatment of skin aging. In recent years, there has been an increasing emphasis on minimally invasive treatments and techniques designed to treat volume loss, wrinkles, and skin photodamage.
Derming, Clinical Research and Bioengineering Institute, viale Cesare Battisti 38, 20900, Monza, Italy

Tel +39039329666

Email adele.sparavigna@derming.com 
Age-related changes in the dermis can be considered the result of intrinsic factors and the consequence of environmental damage, in particular ultraviolet (UV) radiation from the sun, which is responsible for skin photoaging. Both are cumulative processes that share common cellular and molecular pathways mediating skin damages, for example, reactive oxygen species (ROS) arising from oxidative cell metabolism. ${ }^{1}$

The alterations of the dermal connective tissue, corresponding mainly to a reduction of the extracellular matrix (ECM), are highly responsible for the wrinkling and sagging of the skin since they determine deep modifications in its mechanical properties. Repeated studies have proven that aging processes affect the enzymatic activities related to synthesis, remodeling, and catabolism of the ECM components of the dermis (collagen, elastin, and glycosaminoglycans). As a result, not only do aging processes induce a reduction of the ECM density but also, its quality is affected; moreover, the less efficient biosynthetic activities make the newly formed collagen more easily attacked by collagenases and metalloproteinases, the key enzymes in matrix degradation., ${ }^{2,3}$ Today, intradermal injections of biological substances able to induce a revitalization of the dermis can stimulate qualitative and quantitative improvements in aging skin alterations. The most frequently used substance is natural non-cross-linked hyaluronic acid (HA). The levels of HA, the major nonsulfated glycosaminoglycan of the connective tissue scaffold, decrease with aging, an event that leads to a direct reduction in water content and skin turgor. Interestingly, injection of simple HA can, not only provide enrichment of one of the main ECM compounds and deep hydration of the skin but also, strongly stimulates fibroblasts, acting on specific receptors (CD44, RHAMM, and ICAM-1) ${ }^{4}$ to synthesize new scaffold compounds. ${ }^{5}$

Therefore, the goals of biorejuvenation are to increase the biosynthetic capacity of fibroblasts, inducing the reconstruction of an optimal physiologic environment; the enhancement of cell activity; hydration; and the synthesis of collagen, elastin, and HA. The desired effect - firm, bright, moisturized skin - can be achieved by microinjections in the superficial dermis of products containing only one active ingredient or "cocktails" of different compounds that are biocompatible and absorbable. ${ }^{6}$

The great versatility of biorevitalization lies in the different biological effects of the injected active substances. The synergy of different functional ingredients can treat skin in a more complete way, acting on various age-related marks caused by both intrinsic and extrinsic aging factors, with a preventive and curative action.

\section{Objective}

Based on the considerations above, this study aimed to investigate the efficacy of an injectable solution containing $32 \mathrm{mg}$ of nonreticulated HA of biotechnological origin plus an antiaging complex composed of nucleotides, amino acids, vitamins, and antioxidants (Table 1), in the treatment of skin aging and photoaging. The product (Viscoderm ${ }^{\circledR}$ Skinkò E) was commercially available (5 $\mathrm{mL}$ vial) and manufactured by IBSA Farmaceutici Italia Srl (Lodi, Italy). Patients underwent a cycle of four sessions of biorevitalization that was performed in different areas affected by skin aging, and a phototest was performed on dorsal skin, according to the COLIPA method, ${ }^{7}$ to assess the possible photoprotective action of the study product.

\section{Methods}

A total of 64 female healthy volunteers, aged between 37 and 60 years (average age 52 years), were enrolled in this open clinical trial. Inclusion criteria were: patients in good health; accepting to maintain their current eating habits, physical activity, makeup use, and facial cleansing; and accepting to avoid strong UV radiation throughout the entire study period. Exclusion criteria for the study were: subjects who had undergone other medical aesthetical treatments during the previous 3 months; and pregnancy or breastfeeding. All subjects gave written consent for enrollment into the trial.

The objective of the study was to evaluate the tolerability and the antiaging and photoprotective efficacy of an injectable intradermal solution. The study was divided in two different parts: a separate single treatment and UVB

\section{Table I Formulation characteristics}

\begin{tabular}{ll}
\hline Hyaluronic acid & 700 kDa non-cross-linked hyaluronic acid \\
Inorganic salts & Ammonium molybdate, ammonium \\
& metavanadate, calcium chloride, iron sulfate, \\
& potassium chloride, copper sulfate, magnesium \\
chloride, manganese sulfate, sodium acetate, & sodium hydrogen carbonate, sodium chloride, \\
& sodium hydrogen phosphate, sodium metasilicate, \\
& sodium selenite, tin chloride, zinc sulfate \\
& Alanine, arginine, asparagine, aspartic acid, \\
& cysteine, glutamine, glutamic acid, glycine, \\
Amino acids & histidine, isoleucine, leucine, lysine, methionine, \\
& phenylalanine, proline, serine, threonine, \\
& tryptophan, tyrosine, valine, adenine \\
Biotin, calcium pantothenate, choline chloride, & folic acid, nicotinamide, pyridoxine, riboflavin, \\
thiamine, cyanocobalamin & Lipoic acid \\
Inositol, glucose, putrescine, sodium pyruvate
\end{tabular}


exposure to evaluate photoprotective effects, followed by a cycle of four biorevitalizing treatments to examine overall effects. The study design was evaluated and approved by an independent ethics committee. Because of the high number of injections required by this esthetic procedure, the Derming Institues Independent Ethical Committee did not approve the use of a control group treated with placebo, except for the phototest.

\section{Biorevitalization treatment}

A total of five visits were carried out during the trial: at baseline (T0) and after 3 weeks, 6 weeks (T6W), 9 weeks (T9W), and 12 weeks (T12W). Each volunteer underwent four sessions of biorevitalization, one every 3 weeks starting from $\mathrm{T} 0$. The sessions involved multiple microinjections, with either a $0.40 \times 4 \mathrm{~mm} / 27 \mathrm{G}$ or a $0.33 \times 12 \mathrm{~mm} / 23 \mathrm{G}$ needle, in the face (external corner of the eye and cheek), neck, décolletage, and back of the hands. The amount of product injected corresponded to $3 \mathrm{~mL}$ on the face and $5 \mathrm{~mL}$ divided on the other treated areas; the microinjections were performed at a distance of 1-2 $\mathrm{cm}$ from each other.

The assessment of the esthetic result was carried out at $\mathrm{T} 6 \mathrm{~W}, \mathrm{~T} 9 \mathrm{~W}$, and $\mathrm{T} 12 \mathrm{~W}$ and involved the use of clinical evaluations and instrumental measurements (using techniques previously described in the literature), ${ }^{7-18}$ supported by photographic documentation. Visual evaluations were performed monolaterally on the right or left side, according to a randomization list defined by the investigator before the subjects' inclusion, except for the décolletage, which was evaluated in its entirety.

Biorevitalization treatments and clinical evaluations were performed by a dermatologist.

The following instrumental evaluations were performed on the cheek of each volunteer:

a. Tissue dielectric constant values of the superficial and deep skin layers, an index of hydration of the deep skin layers (assessed at $1.5 \mathrm{~mm}$ and $0.5 \mathrm{~mm}$ of depth) was obtained using a MoistureMeterD (Delfin Technologies, Kuopio, Finland). The MoistureMeterD generates a highfrequency, low-power electromagnetic wave to which the tissue is exposed. The reflected electromagnetic wave is registered by the device, and the obtained value is the dielectric constant, which is proportional to the water content of the measured tissue

b. Skin spectrophotometry was done using a visible-UVinfrared (IR) ( $\lambda$ from 300 to $900 \mathrm{~nm}$ ) spectrophotometer (DH2000; TOP Sensor Systems BV, Eerbeek, the Netherlands), which uses a tungsten halogen lamp and a deuterium lamp compliant to CIE (Commission Internationale de l'Éclairage [International Commission on Illumination]) standards. Lamps were switched on 30 minutes before instrument use, in order to stabilize the lamp emissions. The inclination of the probe was $90^{\circ}$ on the surface to be examined, on an area of approximately $2 \mathrm{~mm}^{2}$. The wavelength range was 380-780 nm, corresponding to the visible spectrum

c. Optical colorimetry was performed by a tristimulus colorimeter (Chroma Meter CR-200 ${ }^{\circledR}$; Konica Minolta, Osaka, Japan) equipped with three special filters to obtain red-green-blue (RGB) values in accordance with CIE. The CIE L*a*b* system (CIELAB) is the most complete color space specified by the CIE (1976). ${ }^{19}$ It describes all the colors visible to the human eye. The three coordinates of $\mathrm{L}^{*} \mathrm{a} * \mathrm{~b} *$ represent the lightness of the color $(\mathrm{L} *=0$ yields black, and $\mathrm{L}^{*}=100$ indicates diffuse white; specular white may be higher), its position between red/magenta and green $\left(a^{*}\right.$ negative values indicate green, while positive values indicate magenta), and its position between yellow and blue $\left(b^{*}\right.$ negative values indicate blue, and positive values indicate yellow). Following CIELAB, it was possible to assess skin color: the coordinate $\mathrm{L}^{*}$ defined skin brightness; $a^{*}$ denoted skin erythema; and $b^{*}$ indicated the skin pigmentation

d. Wrinkles pictures were taken and profilometry was done. A picture of the malar region was taken using a Primos compact portable device (GFMesstechnik GmbH, Teltow, Germany). The device software is able to elaborate threedimensional (3D) representations of skin wrinkles, to measure skin principal profilometric parameters, and to directly compare the obtained images. As a measuring method, the Primos compact uses a digital stripe projection based on micromirrors. The portable probe assures a constant distance from the skin as well as a fixed illumination angle of incidence; in this way, it is possible to acquire standardized and reproducible images. By defining an area within the image and tracing a segment of known length in a defined position across the wrinkle and perpendicular to it, it was possible to calculate the

Table 2 Neck wrinkles: severity rating scale

\begin{tabular}{ll}
\hline Score & Description \\
\hline 0 & Absent (no wrinkles) \\
1 & Slight wrinkles \\
2 & Quite evident wrinkles \\
3 & Evident wrinkles \\
4 & Marked wrinkles \\
5 & Very marked wrinkles \\
\hline
\end{tabular}


Table 3 Décolletage and hand surface microrelief regularity scale

\begin{tabular}{ll}
\hline Degree & Description \\
\hline I (very regular) & $\begin{array}{l}\text { The primary lines present all the same depth. The } \\
\text { secondary lines are well demarcated and appear star- } \\
\text { like (apexes converge on several triangles) }\end{array}$ \\
\hline $\begin{array}{l}\text { Hiding and loss of secondary line demarcations. } \\
\text { Star-like pictures are still present but with less }\end{array}$ \\
$\begin{array}{l}\text { demarcated secondary lines } \\
4 \text { (irregular) }\end{array}$ & $\begin{array}{l}\text { Primary line irregularity. Strong hiding of lines with } \\
\text { low presence of star-like appearance } \\
\text { Strong deterioration in the skin. Deep primary line } \\
\text { distortion and loss of secondary lines }\end{array}$ \\
\hline
\end{tabular}

profilometric roughness parameters: average roughness of the analyzed profile; total wrinkle height; and maximum wrinkle depth.

At T0, and at 6, 9, and 12 weeks after the start of treatment, frontal and lateral $\left(45^{\circ}\right)$ pictures of treated areas were taken for each volunteer.

Clinical evaluations of each treated area were performed after the esthetic procedure and were recorded as follows:

a. Skin roughness of the periocular area was assessed according to Glogau's reference photographic scale, ${ }^{20}$ giving a visual score from $0=$ no wrinkles to $3=$ very marked wrinkles

b. Cheek ptosis was assessed according to the Facial Volume Loss Scale, ${ }^{21}$ giving a visual score from 0 to 5

c. Neck wrinkles were assessed according to our internal reference clinical scale, from $0=$ no wrinkles to $5=$ very marked wrinkles (Table 2)

d. Décolletage and hand surface microrelief regularity grade was assessed according to our internal reference clinical scale, from $1=$ very regular to $4=$ very irregular (Table 3 ).

\section{Phototest}

In basal conditions, before starting the aesthetic procedure with the test product, each volunteer will be exposed at the level of dorsal skin to six incremental doses of UVR (ultraviolet radiations) in order to determine the MED (minimal erythema dose of unprotected skin). To determine the protective efficacy of the tested product the UVR irradiation will be repeated 24 hours after a single anti-aging complex treatment performed on the small skin area of the back adjacent to the area where the basal UVR irradiation was performed. The induced erythema was graded $24 \pm 4$ hours after irradiation, according to the COLIPA reference visual score: $0=$ no erythema, $1 / 2=$ perceptible redness reaction (MED), 1= moderate erythema, $2=$ severe erythema. Visual scores of every response to MED-testing were then compared with the ones obtained by irradiated skin previously injected with the study product and irradiated skin previously injected with a placebo (physiological solution for injection). UVB irradiation corresponding to 1 MED was performed 24 hours after the study product/placebo microinjection, and clinical evaluation of the induced erythema was scored $24 \pm 4$ hours after irradiation. The photoprotective efficacy was expressed as a percentage of erythema visual score variation vs placebo.

\section{Statistical analysis}

The statistical evaluations of clinical and instrumental data (adjusted means and standard deviation) and relative graphs were produced using software provided with the statistics manual "Primer of Biostatistics", ${ }^{22}$ as follows:

a. Clinical data analyses included a comparison of results at different study times vs basal conditions, using the

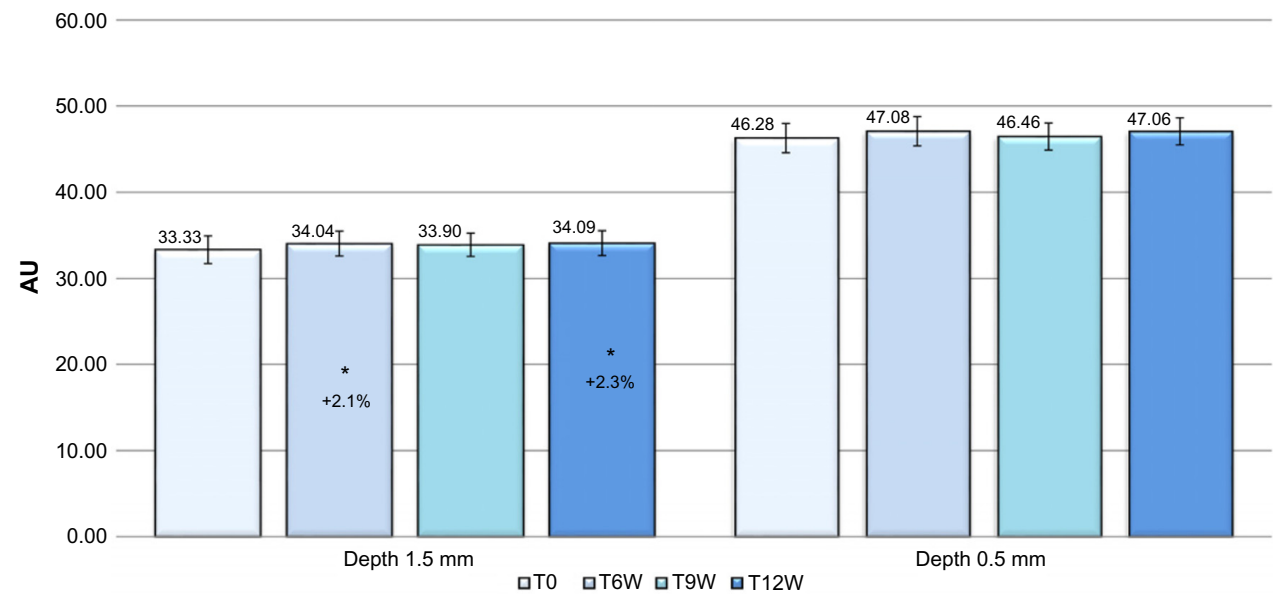

Figure I Skin hydration at 0.5 and $1.5 \mathrm{~mm}$ of depth, variation vs baseline.

Notes: The figure shows measures taken at baseline (T0) and at 6 weeks (T6W), 9 weeks (T9W), and I2 weeks (TI2W) after the start of treatment. Values are expressed as mean value with standard deviation. $* P<0.05$ vs T0, Dunnett test. 
Table 4 Optical colorimetry: variation in L*, a* and b* parameters vs baseline

\begin{tabular}{llll}
\hline & T6W & T9W & TI2W \\
\hline Variation vs baseline \% & & & \\
L* parameter (skin brightness) & $+2 \%^{*}$ & $+2,3 \%^{*}$ & $+2,2 \%^{*}$ \\
a* parameter (skin redness) & $-3,8 \%$ & $-5,5 \%^{*}$ & $-4,3 \%$ \\
b* parameter (skin pigmentation) & $-3,4 \%^{*}$ & $-4,6 \%^{*}$ & $-7 \%^{*}$ \\
\hline
\end{tabular}

Notes: The table shows measures taken at 6 weeks (T6W), 9 weeks (T9W), and 12 weeks $(T I 2 W)$ after the start of treatment. *Dunnett test $P<0.05$.

Friedman test followed by, in case of statistically significant result, the Dunnett test

b. Instrumental data analyses included a comparison of results at different study times vs basal conditions, using analysis of variance (ANOVA) for repeated-measures followed by, in case of statistically significant result, the Dunnett/Tukey test.

\section{Results}

There were five dropouts from the study: subject 47 , for an adverse event related to the second injection procedure; subject 63 for protocol violation (UV light exposure); and subjects 45,52 , and 62 for personal problems unrelated to the study treatment. Therefore the statistical analysis was performed on the total of 59 subjects who completed the study.

\section{Tolerability}

Only one adverse event occurred during the trial: on the day after the second injection procedure, one subject showed edema on the lower eyelids, more marked on the right side, which resolved a few days after the application of an antiinflammatory cream. To assess the relationship between the study product and the event, a small quantity was then injected intradermally on the right forearm of the patient. The redness disappeared normally within 3-4 hours, demonstrating that the adverse event was probably related to the particular sensitivity of the skin area in which the product was injected. At the end of the trial, the investigator judged the product tolerability good-excellent in $100 \%$ of subjects, as confirmed also by the subject self-assessments (29\% as good and $71 \%$ as excellent). No adverse events were reported after the end of the trial.

\section{Instrumental evaluations}

The tissue dielectric constant of the deep skin layers (registered at $1.5 \mathrm{~mm}$ of depth), starting from T6W to T12W, showed a statistically significant increase (Dunnett test $P<0.05$ ), of $2.1 \%$ and $2.3 \%$, respectively, vs baseline. No significant variation was found in skin hydration measured at $0.5 \mathrm{~mm}$ of depth (Figure 1).

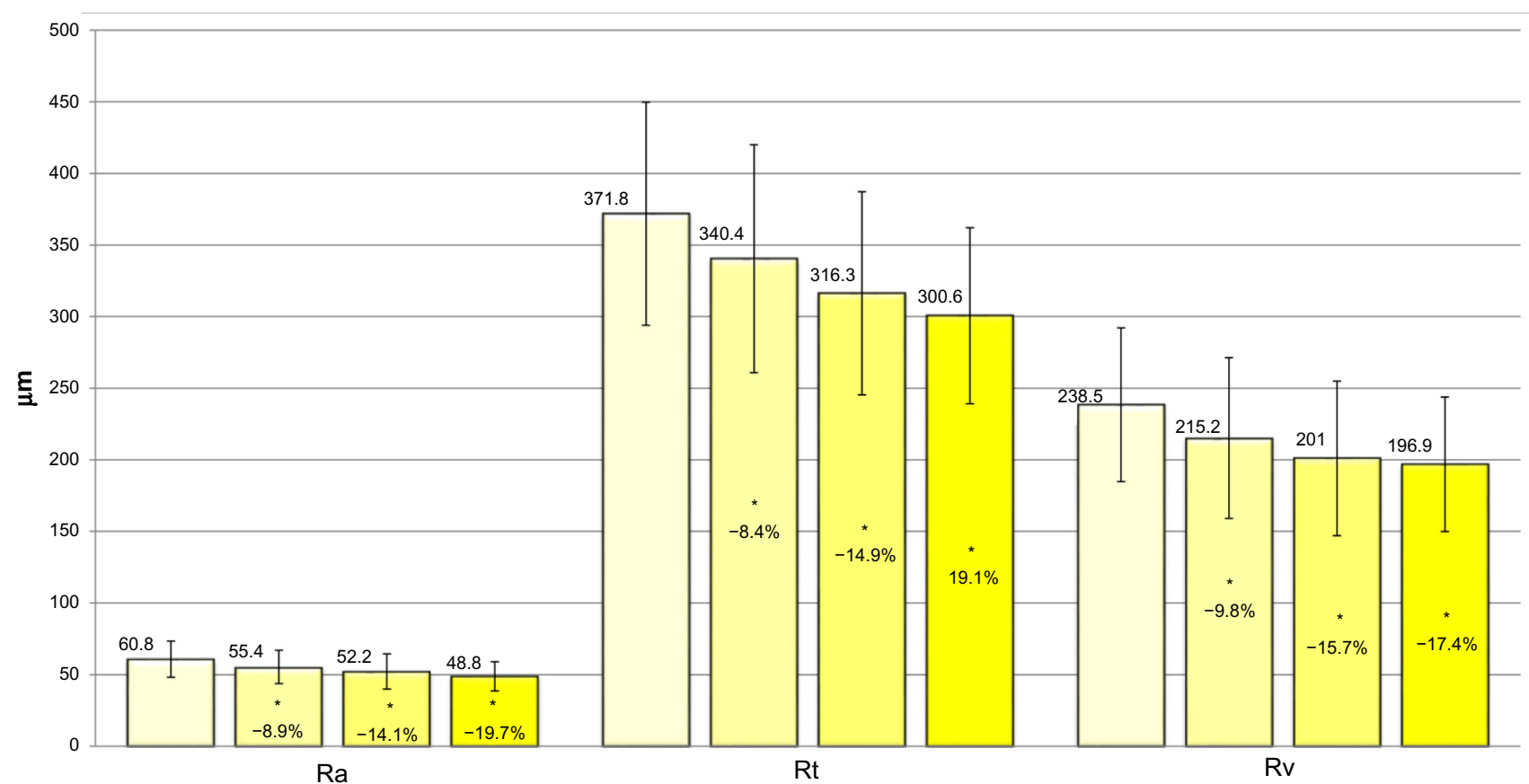

पा0 वT6W वT9W वT12W

Figure 2 Skin profilometric parameters (antiwrinkle efficacy), variation vs baseline.

Notes: The figure shows measures taken at baseline (T0) and at 6 weeks (T6W), 9 weeks (T9W), and I2 weeks (TI2W) after the start of treatment. Values are expressed as mean value with standard deviation. ${ }^{*} P<0.05$ vs T0, Dunnett test.

Abbreviations: Ra, average roughness; Rt, total height; Rv, maximum depth. 


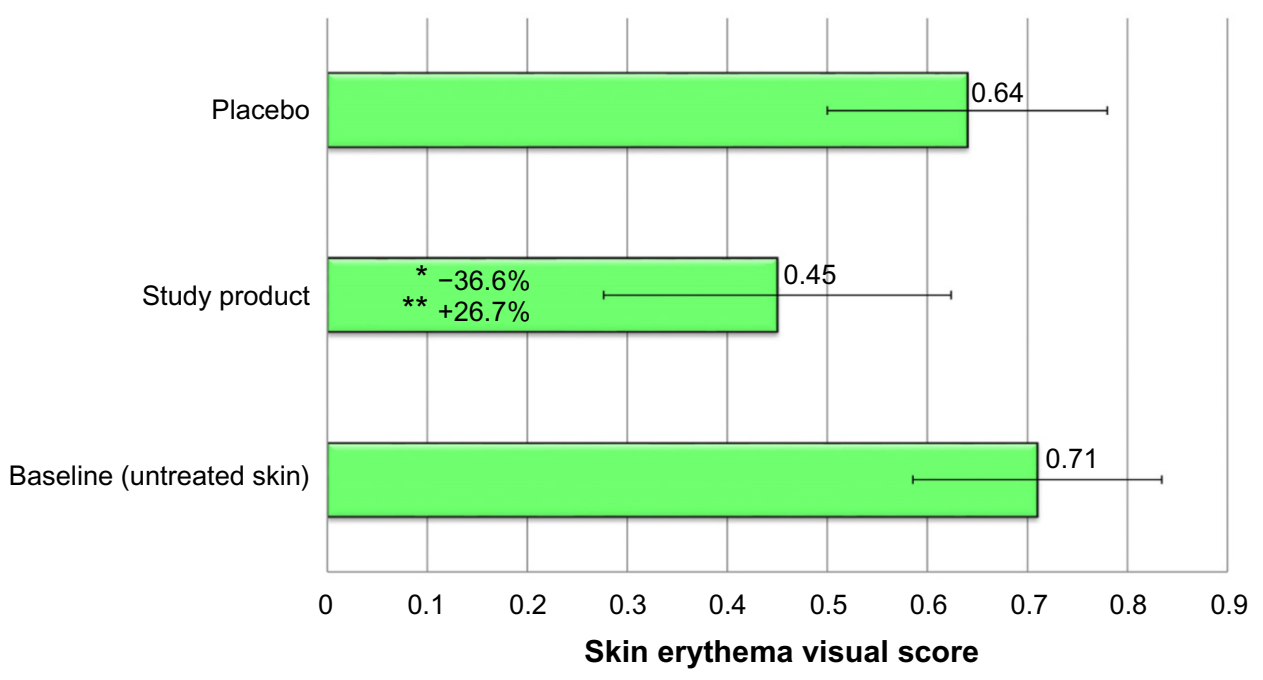

Figure 3 Skin erythema visual score, showing photoprotective efficacy of the study product vs baseline and vs placebo.

Notes: Values are expressed as mean value with standard deviation. $* P<0.05$ vs $T 0$ and $* * P<0.05$ vs placebo, Tukey test.

Spectrophotometry showed, at $\mathrm{T} 9 \mathrm{~W}$ and $\mathrm{T} 12 \mathrm{~W}$, a statistically significant decrease (Dunnett test $P<0.05$ ), of $14.7 \%$ and $15.6 \%$, respectively, of total visible spectrum area vs baseline, suggesting a general improvement of skin face radiance.

Optical colorimetry revealed improvements. The L* parameter data, which indirectly represents the skin brightness, significantly increased starting from the second treatment; moreover, a clinically and statistically significant decrease (Dunnett test $P<0.05$ vs T0) of the a* and $\mathrm{b}^{*}$ parameters, representing, respectively, the skin redness and the skin pigmentation, confirmed the improvement of the face complexion and skin radiance highlighted by the spectrophotometric analysis (Table 4).
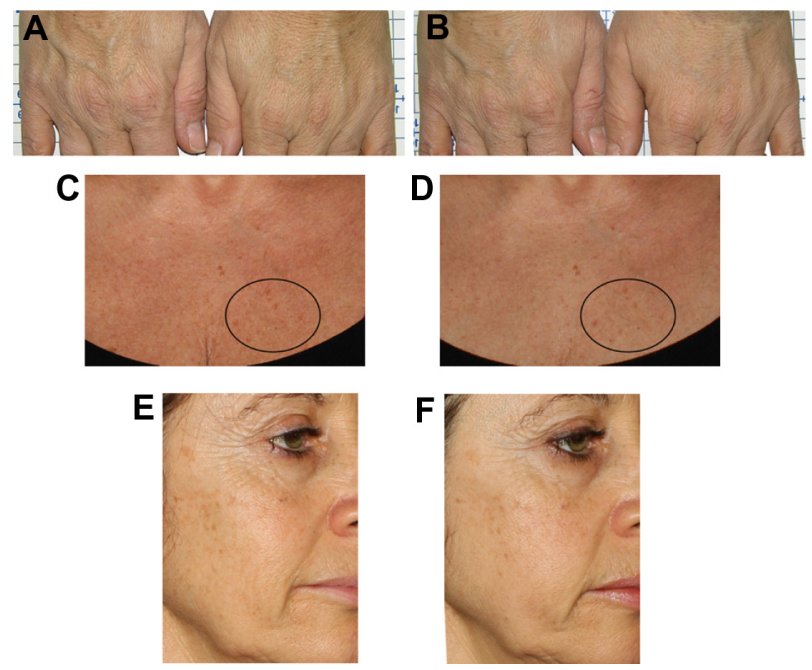

Figure 4 Sample cases.

Notes: Hands at the beginning $(\mathbf{A})$ and at the end $(\mathbf{B})$ of the treatment. Décolletage at the beginning $(\mathbf{C})$ and at the end $(\mathbf{D})$ of the treatment. Face at the beginning $(\mathbf{E})$ and at the end $(\mathbf{F})$ of the treatment.
Image analysis of the periocular area ("crow's feet") showed, as early as the second biorevitalizing treatment, the antiaging efficacy of the tested product. Starting from T6W, evaluations showed a statistically and clinically significant reduction (Dunnett test $P<0.05$, for T6W, T9W, and T12W) of profilometric parameters vs baseline: average roughness of the analyzed profile decreased, by $8.9 \%$ at $\mathrm{T} 6 \mathrm{~W}$, by $14.1 \%$ at $\mathrm{T} 9 \mathrm{~W}$, and by $19.7 \%$ at $\mathrm{T} 12 \mathrm{~W}$, suggesting that the area around the eyes was generally less wrinkled; total wrinkle height decreased, by $8.4 \%$ at $\mathrm{T} 6 \mathrm{~W}$, by $14.9 \%$ at $\mathrm{T} 9 \mathrm{~W}$, and by $19.1 \%$ at $\mathrm{T} 12 \mathrm{~W}$, proving that wrinkles were less deep; and maximum wrinkle depth decreased by $9.8 \%$ at $\mathrm{T} 6 \mathrm{~W}$, by $15.7 \%$ at $\mathrm{T} 9 \mathrm{~W}$, and by $17.4 \%$ at $\mathrm{T} 12 \mathrm{~W}$. These results demonstrate that wrinkles were less visible (Figure 2).

\section{Photoprotective efficacy}

The study product demonstrated a clinically and statistically significant decrease in the erythema visual score compared with baseline $(-36.6 \%)$ (Tukey test $P<0.05$ ). This result highlights a protective efficacy of the study product, statisti-

Table 5 Percentage of subjects, who achieved an improvement of at least one grade from baseline, for each item, at T6W, T9W and $\mathrm{T} I 2 \mathrm{~W}$

\begin{tabular}{lccc}
\hline & T6W & T9W & TI2W \\
\hline \multicolumn{2}{l}{ Percentage of subjects, improvement of at least one grade } \\
Crow's feet roughness & $29 \%$ & $49 \%^{*}$ & $58 \% *$ \\
Cheek ptosis & $8 \%$ & $31 \%^{*}$ & $36 \% *$ \\
Neck wrinkles & $15 \%$ & $31 \%^{*}$ & $34 \% *$ \\
Décolletage surface microrelief & $30 \%$ & $43 \% *$ & $43 \% *$ \\
Hand surface microrelief & $47 \%$ & $62 \% *$ & $60 \% *$ \\
\hline
\end{tabular}

Notes: The table shows measures taken at 6 weeks (T6W), 9 weeks (T9W), and 12 weeks $(T I 2 W)$ after the start of treatment. *Dunnett test $P<0.05$. 
Table 6 Summary table with an overview of the main results at $\mathrm{TI} 2 \mathrm{~W}$

Variation vs baseline \% at TI2W

Deep skin hydration $1.5 \mathrm{~mm} \quad$ Tissue dielectric constant $\quad+2,3 \% *$

of deep skin layers

L* parameter skin brightness

Optical colorimetry

b* parameter skin pigmentation

Optical colorimetry

Phototest

Skin erythema visual score

Clinical evaluation

Clinical evaluation

Hands surface microrelief

Notes: The table shows measures taken at 12 weeks $(\mathrm{T} / 2 \mathrm{~W})$ after the start of treatment. *Dunnett test $P<0.05$.

cally different from the area pretreated with placebo $(-26.7 \%$ Tukey test $P<0.05$ ) (Figure 3). The protective efficacy vs placebo, was calculated using the following formula:

$$
\begin{aligned}
& {\left[\frac{\left(\mathrm{MED}_{\text {product }}-\mathrm{MED}_{\mathrm{T} 0}\right)}{\mathrm{MED}_{\mathrm{T} 0}} \times 100\right]} \\
& -\left[\frac{\left(\mathrm{MED}_{\text {placebo }}-\mathrm{MED}_{\mathrm{T} 0}\right)}{\mathrm{MED}_{\mathrm{T} 0}} \times 100\right]=26.7 \%
\end{aligned}
$$

\section{Clinical evaluations and photographic documentation}

The antiaging efficacy was also confirmed by clinical evaluations. Results highlighted, starting from T9W (after the third biorevitalizing session), a clinically and statistically significant improvement (Dunnett test $P<0.05$, at T9W and $\mathrm{T} 12 \mathrm{~W}$ ) of at least one grade of the following items vs baseline: crow's feet roughness, cheek ptosis, neck wrinkles (rings of Venus), décolletage, and hands skin surface microrelief. In particular, as soon as after the second biorevitalizing treatment, an improving trend in hand skin microrelief was highlighted in $47 \%$ of volunteers. The percentage of subjects who achieved an improvement of at least one grade from baseline for each item at $\mathrm{T} 6 \mathrm{~W}, \mathrm{~T} 9 \mathrm{~W}$, and $\mathrm{T} 12 \mathrm{~W}$ are summarized in Table 5.

A surprising reduction in senile lentigines on the treated areas was observed in several volunteers, as reported by photographic documentation (Figure 4).

\section{Conclusion}

Obtained data showed that the study product provided clinical and biophysical changes in the skin. Interesting quantitative and qualitative results were shown, especially, a multifunctional product activity. Thanks to its rich formulation, the injectable solution of hyaluronic acid plus an antiaging complex (Viscoderm ${ }^{\circledR}$ Skinkò E) demonstrated its effectiveness in the treatment of skin aging, confirmed by all the instrumental evaluations. The analysis of the profilometric parameters showed a statistically and clinically significant reduction of skin roughness around the eyes, as soon as after the second biorevitalizing treatment. The instrumental evaluations were confirmed by clinical evaluations (Table 6). In fact, compared with baseline, skin appeared younger after the third session, and a clinically and statistically significant improvement of at least one grade of crow's feet and cheek ptosis was registered. The efficacy of the study product was observed, not only on the face but also, in the other treated skin areas, confirming the study product versatility. Moreover, an important reduction of the rings of Venus on the neck was highlighted, as was a relevant improvement of the décolletage and hand skin surface microrelief (an index of a redensifying activity).

The study confirmed the well-known efficacy of intradermal injections of HA on skin rejuvenation, which may have increased the biosynthetic capacity of fibroblasts and the synthesis of collagen, elastin, and HA in treated areas. It is generally assumed that when injected intradermally with others active ingredients, HA promotes fibroblasts to express collagen type 1, matrix metalloprotease, and tissue inhibitor of matrix metalloprotease- $1,{ }^{23}$ gaining as a final effect, a skin renewal. Amino acids and vitamins are also important ingredients in a mesotherapeutic cocktail, thanks to properties essential for maintaining a youthful appearance. As documented, the vitamin B complex (vitamins B1 [thiamine], B2 [riboflavin], B5 [pantothenate], B6 [pyridoxine], B9 [folic acid], and B12 [cyanocobalamin]) plays a fundamental role in several metabolic processes, acting as free radical scavengers. Also important are vitamins $\mathrm{H}$ (biotin) and I (inositol), and the amino acids, which take part in the synthesis of polypeptides, forming the matrix of the cellular architecture. ${ }^{24}$

More interesting, skin hydration results showed surprising data: assessment of skin hydration on the cheeks, in particular, showed a positive difference in the percentage hydration of deep and superficial skin layers at the end of the trial, suggesting improved skin moisture retention of the stratum corneum. The study product could have acted as a water content modulator in skin layers, thus improving the epidermal barrier function often affected by the aging mechanism.

In addition to the already known antiaging activity of intradermal injections of HA, Viscoderm ${ }^{\circledR}$ Skinkò E showed novel properties, such as photoprotective efficacy and activity on complexion homogeneity.

The study product showed a substantial protection against damage caused by 1 MED UVB exposure: skin erythema in the pretreated areas was significantly less marked than in untreated or placebo treated skin. This is related to the antioxidant activity of the ingredients, including lipoic acid. 
Lipoic acid is a powerful antioxidant within the cells and at the same time, a coenzyme that participates in complex reactions of cellular metabolism. It plays an important role as a topical photoprotectant thanks to its interesting antioxidant property; in fact, its use is proposed both for treatment of cutaneous aging and for the prevention of the erythema associated with exposure to UV radiation. ${ }^{25}$

Spectrophotometric and optical colorimetric measurements showed an illuminating and brightening activity as soon as after the second treatment session, while the photographic documentation highlighted a clinically relevant and interesting reduction of senile lentigines in the treated areas of some volunteers. These hyperpigmented macules are a common component of photoaged skin, ${ }^{26}$ caused by ROS, which deplete and damage nonenzymatic and enzymatic antioxidant defense systems. ${ }^{27}$ This last evidence suggests that the injected product could intervene at different moments of the skin pigmentation process by activating an intrinsic photoprotective mechanism and improving skin pigmentation quality, with a clearing action on melanin aggregates. It is possible these processes employ common mechanisms, in which antioxidants could play a pivotal role. This last hypothesis deserves further investigation.

\section{Disclosure}

The study was supported by IBSA Farmaceutici Italia Srl, the company that distributes the product used in the trial. The authors report no other conflicts of interest in this work.

\section{References}

1. Peres PS, Terra VA, Guarnier FA, Cecchini R, Cecchini AL. Photoaging and chronological aging profile: Understanding oxidation of the skin. J Photochem Photobiol B. 2011;103(2):93-97.

2. Varani J, Dame MK, Rittie L, et al. Decreased collagen production in chronologically aged skin: roles of age-dependent alteration in fibroblast function and defective mechanical stimulation. Am J Pathol. 2006;168(6):1861-1868.

3. West MD. The cellular and molecular biology of skin aging. Arch Dermatol. 1994;130(1):87-95.

4. Entwistle J, Hall CL, Turley EA. HA receptors: regulators of signalling to the cytoskeleton. J Cell Biochem. 1996;61(4):569-577.

5. Ghersetich I. Management of aging skin. J Eur Acad Dermatol Venereol. 1997;9:51. Abstract.

6. Iorizzo M, De Padova MP, Tosti A. Biorejuvenation: theory and practice. Clin Dermatol. 2008;26(2):177-181.

7. Enterprise and Industry Directorate-General. Standardisation Mandate Assigned to CEN Concerning Methods for Testing Efficacy of Sunscreen Products. Brussels: European Comission; 2006. Available from: http:// ec.europa.eu/consumers/sectors/cosmetics/files/doc/sunscreen_mandate_ en.pdf. Accessed December 31, 2014.
8. Rieger MM, Battista GW. Some experiences in the safety testing of cosmetics. J Soc Cos Chem. 1964;15(3):161-172.

9. wma.net [homepage on the Internet]. WMA declaration of Helsinki. Ethical principles for medical research involving human subjects. World Medical Association; 1964 (updated 2013). Available from: http://www. wma.net/en/30publications/10policies/b3/. Accessed April 11, 2014.

10. Mayrovitz HN, Bernal M, Brlit F, Desfor R. Biophysical measures of skin tissue water: variations within and among anatomical sites and correlations between measures. Skin Res Technol. 2013;19(1):47-54.

11. Mayrovitz HN, Bernal M, Carson S. Gender differences in facial skin dielectric constant measured at $300 \mathrm{MHz}$. Skin Res Technol. 2012; 18(4):504-510.

12. Takiwaki H. Measurement of skin color: practical application and theoretical considerations. J Med Invest. 1998;44(3-4):121-126.

13. Fullerton A, Fischer T, Lahti A, Wilhelm KP, Takiwaki H, Serup J. Guidelines for measurement of skin colour and erythema. A report from the Standardization Group of the European Society of Contact Dermatitis. Contact Dermatitis. 1996;35(1):1-10.

14. Westerhof W. CIE Colorimetry. In: Serup J, Jemec GBE, editors. Handbook of Non-Invasive Methods and the Skin. Boca Raton, FL: CRC Press. 1995;385-397.

15. Frankowsky G, Hainich R. DPL-based 3D metrology by structured light or projected fringe technology for life sciences and industrial metrology. In: Hornbeck LJ, Douglas MR, editors. Proc SPIE 7210: Emerging Digital Micromirror Device Based Systems and Applications. Bellingham, WA: SPIE Publications; 2009;1-12.

16. Hoppe U, Sauermann G, Lunderstadt R. Quantitative analysis of the skin's surface by means of digital signal processing. J Soc Cos Chem. 1985;36(2):105-123.

17. Hof C, Hopermann H. Comparison of replica- and in-vivo measurement of the microtopography of human skin. SOFW Journal. 2000;126: $40-47$.

18. Berger DS. Specification and design of solar ultraviolet simulators. J Invest Dermatol. 1969;53(3):192-199.

19. Baldevbhai PJ, Anand RS. Color image segmentation for medical images using L*a*b* color space. IOSR Journal of Electronics and Communication Engineering. 2012;1(2):24-45.

20. Glogau RG. Aestethic and anatomic analysis of the aging skin. Semin Cutan Med Surg. 1996;5(3):134-138.

21. Ascher B, Coleman S, Alster T, et al. Full scope of effect of facial lipoatrophy: a framework of disease understanding. Dermatol Surg. 2006;32(8):1058-1069.

22. Glantz SA. Primer of biostatistics. 5 th ed. McGraw-Hill, Medical Pub. Div.; 2002.

23. Gao F, Liu Y, He Y, et al. Hyaluronan oligosaccharides promote excisional wound healing through enhanced angiogenesis. Matrix Biol. 2010;29(2):107-116.

24. Tosti A, De Padova MP. Atlas of Mesotherapy in Skin Rejuvenation. London: Informa Healthcare; 2007.

25. Bast A, Haenen GR. Lipoic acid: a multifunctional antioxidant. Biofactors. 2003;17(1-4):207-213.

26. Situm M, Bulat V, Buljan M, Puljiz Z, Situm V, Bolanca Z. Senile lentigo - cosmetic or medical issue of the elderly population. Coll Antropol. 2010;34 Suppl 2:S85-S88.

27. Wlaschek M, Tantcheva-Poór I, Naderi L, et al. Solar UV irradiation and dermal photoaging. J Photochem Photobiol B. 2001;63(1-3):41-51. 


\section{Publish your work in this journal}

Clinical, Cosmetic and Investigational Dermatology is an international, peer-reviewed, open access, online journal that focuses on the latest clinical and experimental research in all aspects of skin disease and cosmetic interventions. All areas of dermatology will be covered; contributions will be welcomed from all clinicians and basic science researchers globally. This journal is indexed on CAS. The manuscript management system is completely online and includes a very quick and fair peer-review system, which is all easy to use. Visit http://www.dovepress.com/testimonials.php to read real quotes from published authors. 